\title{
ON A THEOREM OF FLANDERS
}

\author{
ROBERT E. HARTWIG
}

\begin{abstract}
It is shown that if $R$ is a regular strongly-pi-regular ring, then $R$ is unit-regular precisely when $(a b)^{d} \approx(b a)^{d}$ for all $a, b \in R$. This generalizes a result by Flanders, which states that the matrices $A B$ and $B A$ over a field $F$ have the same elementary divisors except possibly those divisible by $\lambda$.
\end{abstract}

1. Introduction. A classic theorem of Flanders [3] states that if $A$ and $B$ are $n \times n$ matrices over a field $\mathbf{F}$, then $A B$ and $B A$ have the same elementary divisors, except possibly for those that are powers of $\lambda$.

The purpose of this note is to point out that the real reason why this result is true, is because the matrix ring $\mathbf{F}_{n \times n}$ is both strongly-pi-regular as well as unitregular. We shall use the concept of pseudo-similarity, introduced in [5] to provide the necessary link between strong-pi-regularity and unit-regularity.

We recall that a ring $R$ is called (unit) regular, if for every $a \in R$, there exists a (unit) solution $x \in R$, to the equation $a x a=a$. Such solutions will be denoted at $a^{-}$. A ring $R$ is strongly-pi-regular, $\mathrm{s} \pi \mathrm{r}$ for short, if for every $a \in R$, there is a solution to the equations

$$
a^{k} x a=a^{k}, \quad x a x=x, \quad a x=x a,
$$

for some $k \geq 0$. The solution is unique and is called the Drazin inverse $a^{d}$ of $a$ [2]. In the special cases where $k=0$ or $k=1, a^{d}$ is called the group inverse of $a$, and is denoted by $a^{\#}$. It is well known that the ring $\mathbf{F}_{n \times n}$ of $n \times n$ matrices over a field $\mathbf{F}$ is both $\mathrm{s} \pi \mathrm{r}$ and unit-regular. Two ring elements are called pseudo-similar if

$$
x^{-} a x=b, \quad x b x^{-}=a, \quad x x^{-} x=x
$$

for some $x, x^{-} \in R$. It was shown in [6], that for a unit-regular ring, similarity $(\approx)$ and pseudo-similarity $(\approx)$, coincide. Two idempotents $e$ and $f$ in $R$, are said to be equivalent, $e \sim f$, if $e R$ and $f R$ are isomorphic ( $\cong$ ) as $R$-modules. This may be rewritten as $e \sim f$ if $e=p^{+} p, f=p p^{+}$, for some $p, p^{+} \in R$, that satisfy $p p^{+} p=p$ and $p^{+} p p^{+}=p^{+}$. It is easily seen that $e \sim f \Leftrightarrow e \approx f[4]$ and that $\sim$ actually coincides with the classical $D$-relation on semigroups.

2. Main results. Our generalization of the theorem of Flanders is based on the following two main results.

THEOREM 1. Let $R$ be a strongly-pi-regular ring with unity and let $x, y \in R$. Then the following are equivalent:

(i) $x^{d} \approx y^{d}$,

(ii) $x^{2} x^{d} \approx y^{2} y^{d}$.

In which case

Received by the editors July 10, 1980 and, in revised form, December 9, 1981.

1980 Mathematics Subject Classification. Primary 15A21, 15A09.

(c) 1982 American Mathematical Society 0002-9939/81/0000-0789/\$01.75 
(iii) $x x^{d} \approx y y^{d}$ and $\left(x^{d}+1-x x^{d}\right) \approx\left(y^{d}+1-y y^{d}\right)$.

If in addition $R=\mathbf{F}_{n \times n}$, then each of the above is equivalent to

(iv) $x$ and $y$ have the same elementary divisors, except possibly those that are powers of $\lambda$.

PROOF. (i) $\Leftrightarrow$ (ii) This is based on a result of Drazin [2], which states that if $p q=q p$, and $p^{\#}$ exists, then $p^{\#} q=q p^{\#}$. Now if $x^{d} q=q y^{d}$, with $q$ invertible, then because $\left(x^{d}\right)^{\#}=x^{2} x^{d}$, it follows that $x^{2} x^{d} q=q y^{2} y^{d}$. The converse follows also since $\left(p^{\#}\right)^{\#}=p$.

(i) $\Rightarrow$ (iii) Let $x^{d} q=q y^{d}$, and hence by part (ii) $x^{2} x^{d} q=q y^{2} y^{d}$. Now $x x^{d}=$ $\left(x^{2} x^{d}\right) x^{d}$ and so $x x^{d} q=q y y^{d}$. The remaining result is now also clear.

(iii) $\Rightarrow$ (iv) Let $R=\mathbf{F}_{n \times n}$, and suppose that

$$
x=P\left[\begin{array}{cc}
U_{1} & 0 \\
0 & \eta_{1}
\end{array}\right] P^{-1}, \quad y=Q\left[\begin{array}{cc}
U_{2} & 0 \\
0 & \eta_{2}
\end{array}\right] Q^{-1},
$$

are the core-nilpotent decompositions of $x$ and $y$ respectively, with $U_{i}$ invertible and $\eta_{i}$ nilpotent, $i=1,2$. Then

$$
x^{d}=P\left[\begin{array}{cc}
U_{1}^{-1} & 0 \\
0 & 0
\end{array}\right] P^{-1},
$$

and $x x^{d} \approx y y^{d}$ shows that $U_{1}$ and $U_{2}$ have the same size. Next,

$$
\left[\begin{array}{cc}
U_{1}^{-1} & 0 \\
0 & I
\end{array}\right] \approx x^{d}+1-x x^{d} \approx y^{d}+1-y y^{d} \approx\left[\begin{array}{cc}
U_{2}^{-1} & 0 \\
0 & I
\end{array}\right] \text {. }
$$

Hence, on using elementary divisors, it follows that $U_{1}^{-1} \approx U_{2}^{-1}$ and so $U_{1} \approx U_{2}$. This is equivalent to (iv).

(iv) $\Rightarrow$ (i) This is clear from the fact that $x^{d} \approx y^{d} \Leftrightarrow U_{1}^{-1} \approx U_{2}^{-1} \Leftrightarrow U_{1} \approx U_{2}$.

Our second result deals with the relation between $a b$ and $b a$.

THEOREM 2. Let $R$ be a strongly-pi-regular ring with unity, and let $a, b \in R$. Then the following are equivalent:

(i) $R$ is unit-regular, and

(ii) $R$ is regular and $(a b)^{d} \approx(b a)^{d}$ for all $a, b \in R$.

PROOF. (i) $\Rightarrow$ (ii) Cline's formula [1], states that $(x y)^{d}=x(y x)^{d^{2}} y$. If we apply this to $a b$ and $b a$ we may write

$$
(a b)^{d}=a \cdot(b a)^{d} \cdot(b a)^{d} b
$$

and

$$
(b a)^{d}=(b a)^{d} b \cdot(a b)^{d} \cdot a .
$$

Now since $(b a)^{d} b \cdot a \cdot(b a)^{d} b=(b a)^{d} b$, it follows that $(a b)^{d} \approx(b a)^{d}$. Moreover, $a b(a b)^{d} \approx b a(b a)^{d}$ as well. Next, since $R$ is assumed to be unit-regular, we may conclude $\left[6\right.$, p. 453] that semisimilarity implies similarity. Consequently $(a b)^{d} \approx$ $(b a)^{d}$ and $a b(a b)^{d} \approx b a(b a)^{d}$.

(ii) $\Rightarrow$ (i) Based on a result of Vidav [7], it suffices to show that two equivalent idempotents are similar. Suppose therefore that $e$ and $f$ are equivalent idempotents in $R$, and that $e=p^{+} p f=p p^{+}$, with $p p^{+} p=p$ and $p^{+} p p^{+}=p^{+}$. Now since $e^{d}=e$ and $f^{d}=f$, we have $e=e^{d}=\left(p^{+} p\right)^{d} \approx\left(p p^{+}\right)^{d}=f^{d}=f$. Because $R$ is regular, the proof is now complete. 
Corollary 1. If $A, B \in \mathbf{F}_{n \times n}$, then

(i) $(A B)^{d} \approx(B A)^{d}$,

(ii) $(A B)^{2}(A B)^{d} \approx(B A)^{2}(B A)^{d}$, and

(iii) the elementary divisors of $A B$ and $B A$ coincide except possibly for those that are powers of $\lambda$.

Proof. (i) Since $\mathbf{F}_{n \times n}$ is unit-regular and $s \pi r$, this follows from Theorem 2. The remaining two results follow at once from Theorem 1, thereby completing the proof of Flander's Theorem.

Corollary 2. If $A, B \in \mathbf{F}_{n \times n}$, then $A B \approx B A$ if and only if $\operatorname{rank}(A B)^{k}=$ $\operatorname{rank}(B A)^{k}, k=1,2, \ldots$

Proof. The necessity is clear. For the sufficiency, suppose that $A B=x$ and $B A=y$ are given as in (2.1). It then suffices to show that $U_{1} \approx U_{2}$ and $\eta_{1} \approx \eta_{2}$. From Corollary 1 , we recall that $U_{1} \approx U_{2}$ always holds. Lastly, $\operatorname{rank}(A B)^{k}=$ $\operatorname{rank}(B A)^{k}$ implies that $\operatorname{rank}\left(\eta_{1}^{k}\right)=\operatorname{rank}\left(\eta_{2}\right)^{k}$, for all $k=1,2, \ldots$, which is well known to suffice for $\eta_{1} \approx \eta_{2}$.

REMARKS. 1 . The results of Corollary 1 can easily be modified to include the case where $A$ and $B$ are rectangular, by means of adding zeros suitably.

2. The equivalence of the pseudo-similarity and similarity of $(a b)^{d}$ and $(b a)^{d}$, hinges on the existence of a unit solution to the equation $(b a)^{d} b \cdot x \cdot(b a)^{d} b=(b a)^{d} b$. Even though several obvious solutions exist, such as $x=a$ or $x=a+1-(b a)(b a)^{d}$, it is not known whether there exists a unit solution $u$ that can be expressed in terms of $a, b$ and $(\cdot)^{d}$ exclusively.

3. It is not known whether the converse of Theorem 1 holds in general. That is whether $x x^{d} \approx y y^{d}$ and $x^{d}+1-x x^{d} \approx y^{d}+1-y y^{d}$ ensure that $x^{d} \approx y^{d}$.

\section{REFERENCES}

1. R. E. Cline, An application of representations for the generalized inverse of a matrix, MRC Technical report, \#592, 1965.

2. M. P. Drazin, Pseudo-inverses in associative rings and semigroups, Amer. Math. Monthly 65 (1958), 506-514.

3. H. Flanders, Elementary divisors of $A B$ and $B A$, Proc. Amer. Math. Soc. 2 (1951), 871-874.

4. R. E. Hartwig, Unit and unitary solutions to $A X=B$ (submitted).

5. R. E. Hartwig and F. Hall, Pseudo-similarity for matrices over a field, Proc. Amer. Math. Soc. 71 (1978), 6-10.

6. R. E. Hartwig and J. Luh, A note on the group structure of unit regular ring elements, Pacific J. Math. 71. (1977), 449-461.

7. I. Vidav, Modules over regular rings, Math. Balkanica 1 (1971), 287-292.

Department of Mathematics, North Carolina State University, Raleigh, NORTH CAROLINA 27650 